\section{Color Matching to Ink Jet Printers from a Computer Screen, Part 1.}

\author{
Jerry Sedgewick \\ University of Minnesota \\ info@quickphotoshop.com
}

The ability to print what is seen on a computer screen so that it matches a printer (color matching) confounds even the most experienced. That's because computer monitors, even from the same manufacturer, cannot be made to display colors identically. Also, printers from different manufacturers contain several "libraries" of replacement colors in order to replace pixel values in an image from light (red, green and blue: RGB) to the primary colors for pigments (cyan, magenta, yellow and black: CMYK). Cameras use the primary colors for light and that is the way the digital image is stored and displayed; printers use the primary colors for pigments in ink. Thus, a printer must have a pixel by pixel list of replacement CMYK values for every RGB value in any image for that manufacturer's idea of faithful color matching.

It's true that calibration software and hardware can be purchased to aid in color matching. This really helps when a calibration guide consisting of a variety of hues (intermediates of various colors) at known values is printed on typical paper stock, and then the monitor is adjusted to display those calibrated hues correctly. The caveat, however, lies in this limitation: Hues will only be correctly displayed when using that printer and that paper. Each printer and paper combination needs its own calibration guide and several of these are then stored on the computer as .icm files (internal calibration of monitor; also shown as .icc). When that printer and paper combination is chosen, the icm file is loaded in the control panel display settings. That is precisely what is done in many graphic design and advertising agencies in order to get better prediction of color reproduction on paper.

Others have opted to forgo the calibration mess altogether. Instead, they rely upon typical values for a variety of objects (e.g., Caucasian skin), a process that is explained in several publications. Or, they might also rely upon Adobe's color management in Photoshop itself, a process designed to help predict color outcome, depending on the intent (web, for printing, to publisher, etc.). Adobe's color management attempts to display colors within the gamut (range of colors available) of each possible output. The range of colors available from the mixing of red, green and blue when using a camera, for example, is different than the range of colors for cyan, magenta, yellow and black when using a printer; and that will be different than the range of colors displayed on the web.

The limitation to both these methods lie in the nature of images that are printed in research: most colors are saturated or nearsaturated, such as those used in psuedocolor. These are not typical colors in the world of photography. These are not the typical colors seen in family or travel pictures, the kind of photography to which manufacturers and software companies optimize their products.

Color matching is also confounded by a person's ability to accurately interpret the hue, something that requires a training of the eye (accomplished artists, for example, can no longer see the color white; instead, they see reflections of other hues in white). If one color is surrounded by other colors, its hue will be interpreted differently. Hues can be saturated (farthest away from gray: neon colors) or unsaturated, so that the color pink can appear as hot

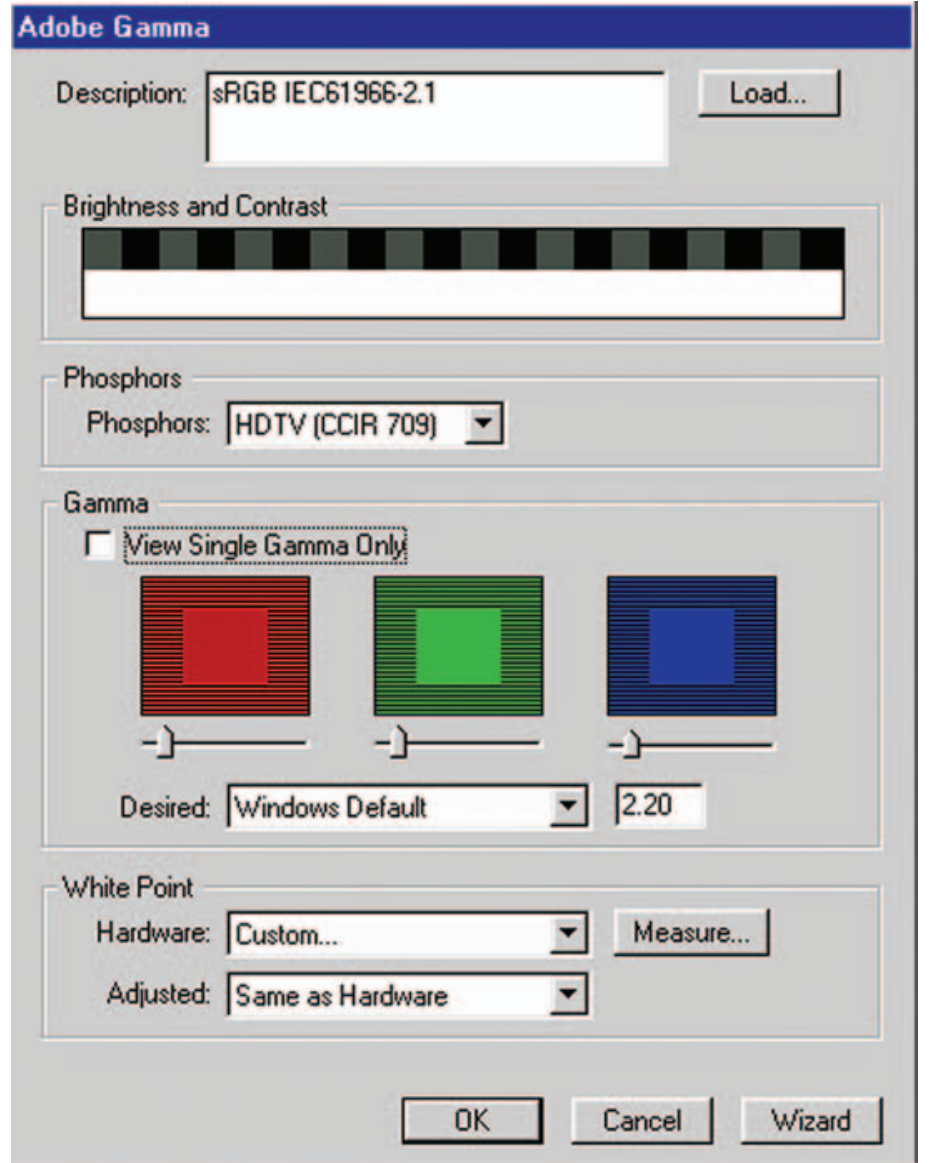

pink or as a subtle grey-pink. The hue can be light or dark, so that a red can be see-through or nearly black. The lighting in the room can affect how colors reflect from a print. These colors need to be close to daylight in order to gain accurate interpretation, or viewing booths need to be purchased with lamps rated to 5500 degrees Kelvin (in simplest terms, what is commonly agreed upon to be closest to middle of the day sunlight). Finally, interpretation is psychological. Both a remembered color exists for any one image which fits what was believed to have been seen, and a color that suggests itself based upon the hypothesis (what a person wishes the color would be). Given all these dynamics, it's no wonder that no two individuals in one room agree upon color, something that can lead to a whole afternoon of print after print.

A basic approach. While no simple approach can solve every color matching dilemma, there are a few things that can be done to improve chances of getting a computer screen to color match a photographic print without a huge outlay of money or time. This approach depends upon the use of a single computer screen devoted to one or two printers. The monitor should be a cathode ray tube (CRT) monitor versus a flat screen because it will be able to display more hues (though this isn't critical). It's best to have a monitor that is not too old, since these lose their ability to display hues correctly over time (a yellowish screen means the monitor should be replaced). The printer(s), if a thermal or photographic printer is not already in use, should be ink jets, mostly because of their cost and cross-ability to print out text and images without the appearance of small dots (such as what is seen on a laser printer; a printer to be avoided, if possible). If one printer is used, then it is best to use an ink jet with 6 cartridges (including black) each of which can be replaced individually. Professional photographers 


\section{in your drive for excellence, get a little extra help...}

Today, it is no longer enough to make leading edge products.

Support, training, and top quality service are key elements of our unique solutions for microanalysis.

Before purchasing your next EDS, WDS or EBSD system, ask for your free guide to 'Microanalysis Explained', and see how Oxford Instruments can boost your drive to success.
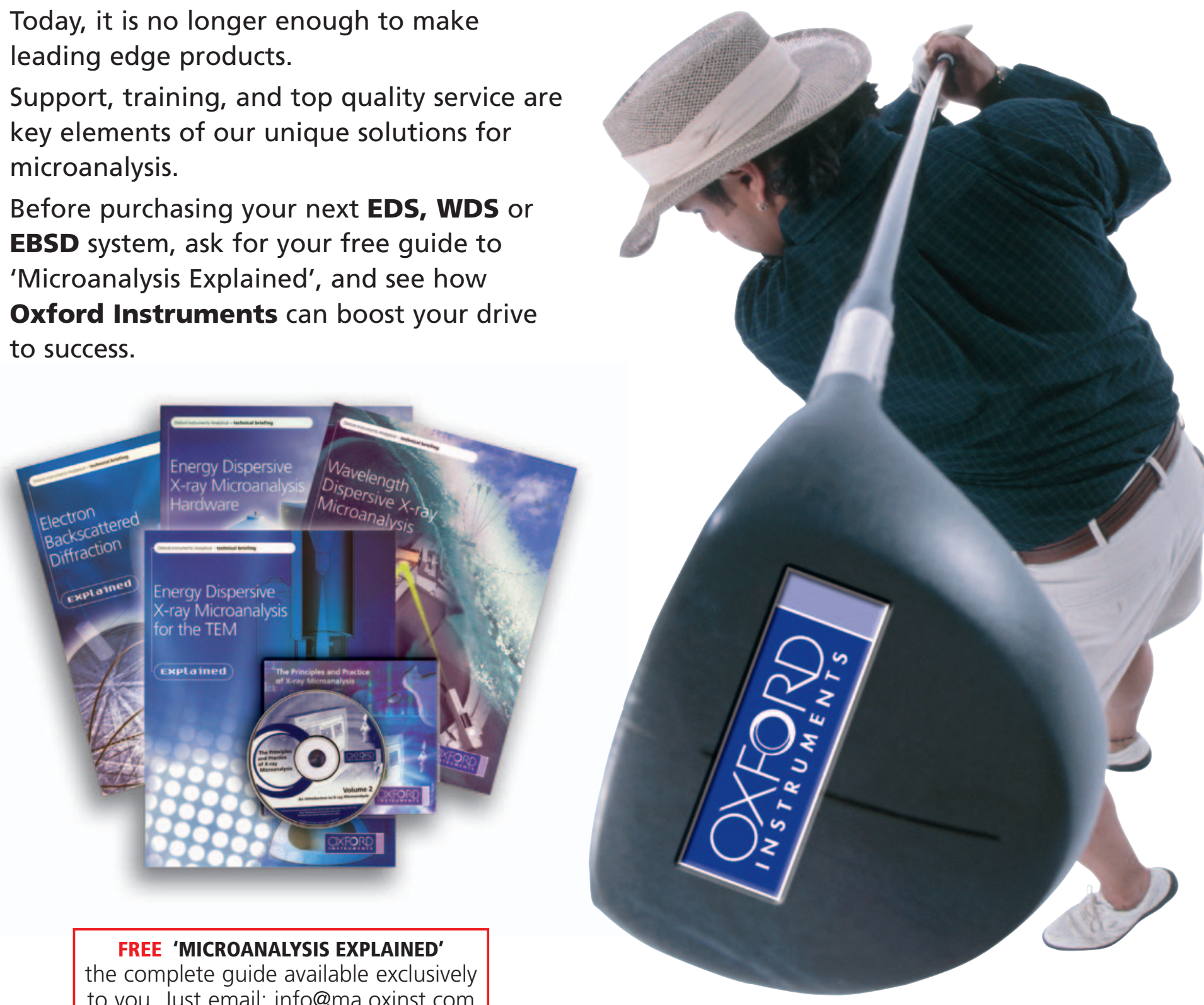

FREE 'MICROANALYSIS EXPLAINED' the complete guide available exclusively to you. Just email: info@ma.oxinst.com

Oxford Instruments Analytical

Concord MA 


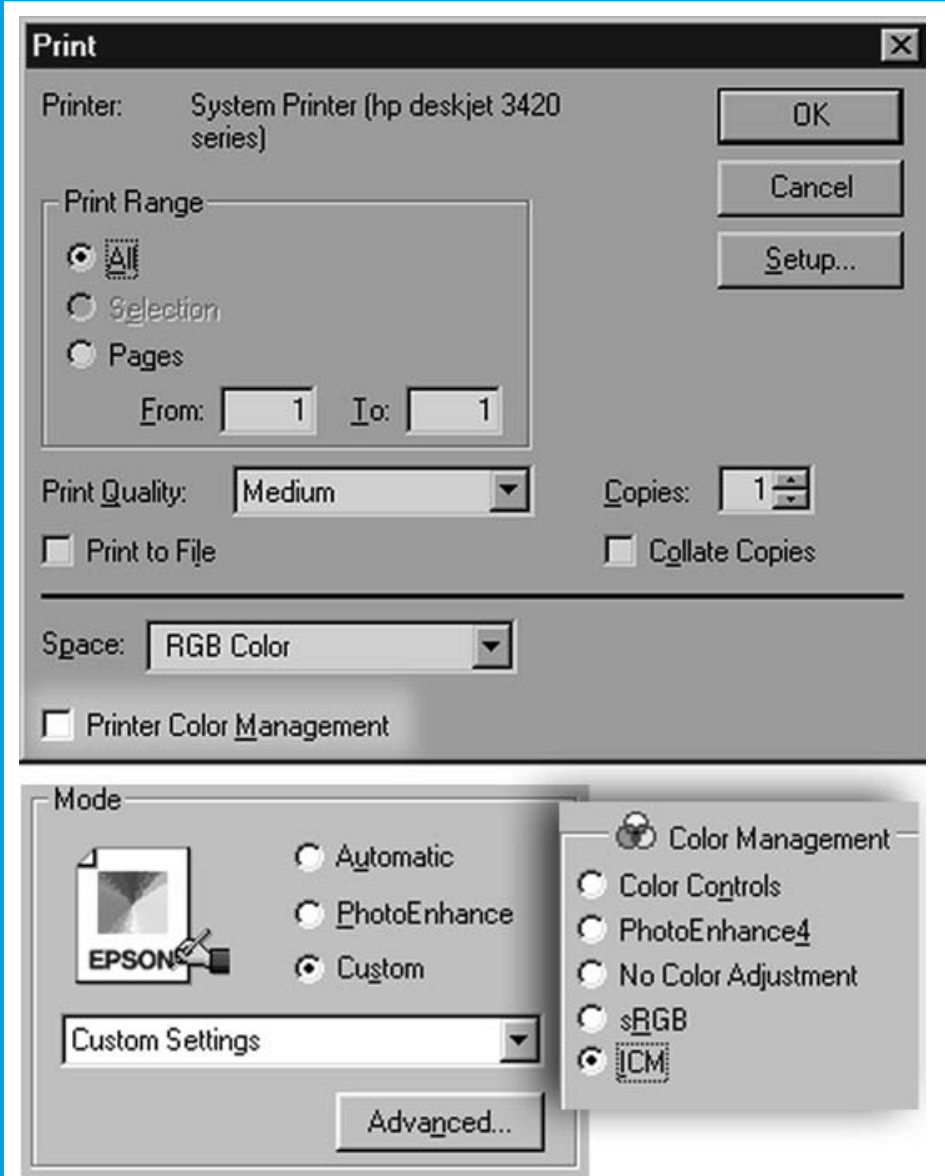

tend toward Epson printers, but that should not be interpreted as what is most useful for those in research, for I have seen side-by-side prints of histology sections that looked better on Hewlett-Packard and Canon ink jets. In any case, it is wise to purchase two ink jets: one for speed and the other for quality, or simply to provide two choices. Be sure to use optimum quality, glossy paper.

Adjust the computer screen. It is, first of all, necessary to set the computer screen to more optimal settings for printing scientific images. Computer monitors need to be in rooms without excessive sunlight coming in through windows, best in rooms that are fairly dark. Proceed with the following steps to optimize the screen and to create a custom. $\mathrm{icm}$ file:

1. Find Adobe Gamma in the control panel of your computer. This program should have been loaded when Photoshop was installed. It is also the program that might have been used when first using Photoshop, when prompted to set gamma. It would be a good idea to set this again, and, perhaps, every 6 months or so.

2. Profile setting. Use either the step-by-step wizard or Control Panel (Fig. 1). Give your profile a specific name rather than using the Load button to load an existing profile.

3. Monitor settings. Turn the contrast up all the way on your monitor using button controls on the monitor itself (or keyboard). Then adjust brightness so that a dark gray box is just visible in the center of a black box within a white frame.

4. Phosphors. Only change these if you know what the settings should be.

5. Single Gamma Only. Uncheck "View Single Gamma Only" and look at red, green and blue channels individually. Blur your vision or take off your glasses and move slider under each until patterns on the inside box match the pattern of the frame. This takes a bit of practice.

6. Set Gamma. Adobe may recommend a gamma of 1.8 in the United States, but, in research, this is better set at 2.2 for higher contrast. Gamma, in the simplest explanation, sets the contrast of midtones in an image, and not so much the whites or darker shadows. The contrast should be higher in order to avoid making blacks too dark, since a) the values at the black end of an image are not discernable by eye, b) dark, shadow areas do not print well and c) deep blacks make it more difficult to create good color matches when going from RGB color to CMYK color (for publication). This setting seems counterintuitive, since deep blacks are appreciated by scientists and the desire is always to increase the deepness of blacks, but printers can only print deep blacks to a certain threshold; any blacker and these cannot be printed.

7. Hardware White Point. Go through the exercise of setting the white point. This, too, takes a bit of judgment, for the most "neutral" gray square must be chosen. This would be a gray that does not shift toward red or green or blue. If the middle square is correct and it is clicked on, then the screen returns to the control panel. If the middle is not chosen at the get go, then more sets of squares are presented until the middle appears correct.

8. Adjusted White Point. Keep the same as Hardware, unless you know what you're doing.

9. Click Finish and save the profile with your custom settings as an .icm file.

Ink Jet Printers. This is a good start for color management, but these settings will not work with standard settings that come with ink jet printers. Software for these printers assumes "real world" photography, and so the deepness of blacks necessary for science cannot be achieved with default printer preferences. Also, the colors are mostly primary, and these, too, become difficult for printers to interpret. Thus, it is best to either turn off any kind of color interpretation on the printer, or to set the printer so that it attempts to imitate what is shown on your computer screen. That decision is best made on one's own through trial and error, but one or the other should be chosen for scientific photos.

Setting the printer (Fig. 2). To set the printer, find Options or Preferences buttons and keep digging in order to find a dialogue box displaying preferences for color management. Choose either "No color management" or "ICM/ICC color management" or similarly worded checkboxes. Make a print with no color management and one with ICM color management to determine which prints best. Make sure to print to high quality, glossy paper. Evaluate your print in daylight coming through windows, or in a viewing booth.

Now you are ready to match colors. In the next issue, this process will be described, as well as Photoshop color setting decisions.

Note: More thorough explanations of the concepts presented in this article can be found in "The Image Processing Handbook" by John Russ.

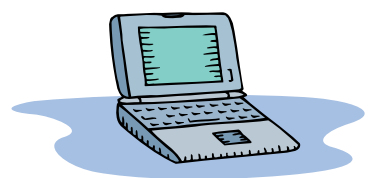




\section{Venture Into The Now}

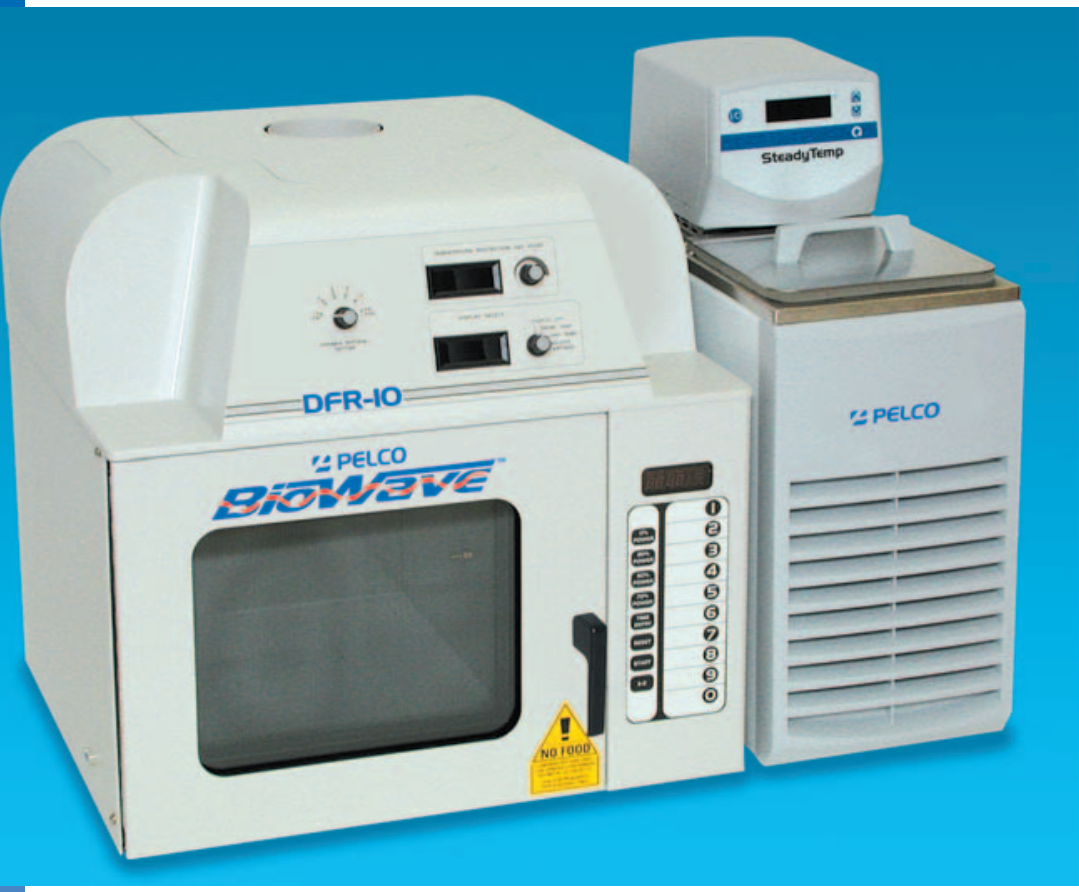

EM \& LM Processing, Immunolabeling, Bone Decalcification: PELCO ${ }^{\circledR}$ Microwave Tissue Processors have more than one use

PELCO BioWave ${ }^{\circledR}$ DFR-10 Laboratory Tissue Processing System

Immunology, Neurosciences, Diagnostic EM, Histology, Botany, Pharmacology and Biology are some of the fields of application. Your routine tissue processing or special investigatory trials invite methods that can improve final results and speed your work.

Variables offered by the PELCO BioWave ${ }^{\circledR}$ models, which may be adjusted to get optimum results are:

\section{Low Temperature Processing}

The only laboratory microwave system that does not rely on heating protocols but rather application of microwave energy with heat removal to optimize results.

\section{True Variable Power}

Several power levels may be selected to give $100 \%$ of that level during a time period; this means full control and constant application of power even at low wattages.

\section{PELCO ColdSpot ${ }^{\circledR}$}

A patented, effective method to hold a flat area in the processor at a certain temperature level. No hot or cold spots to "locate".
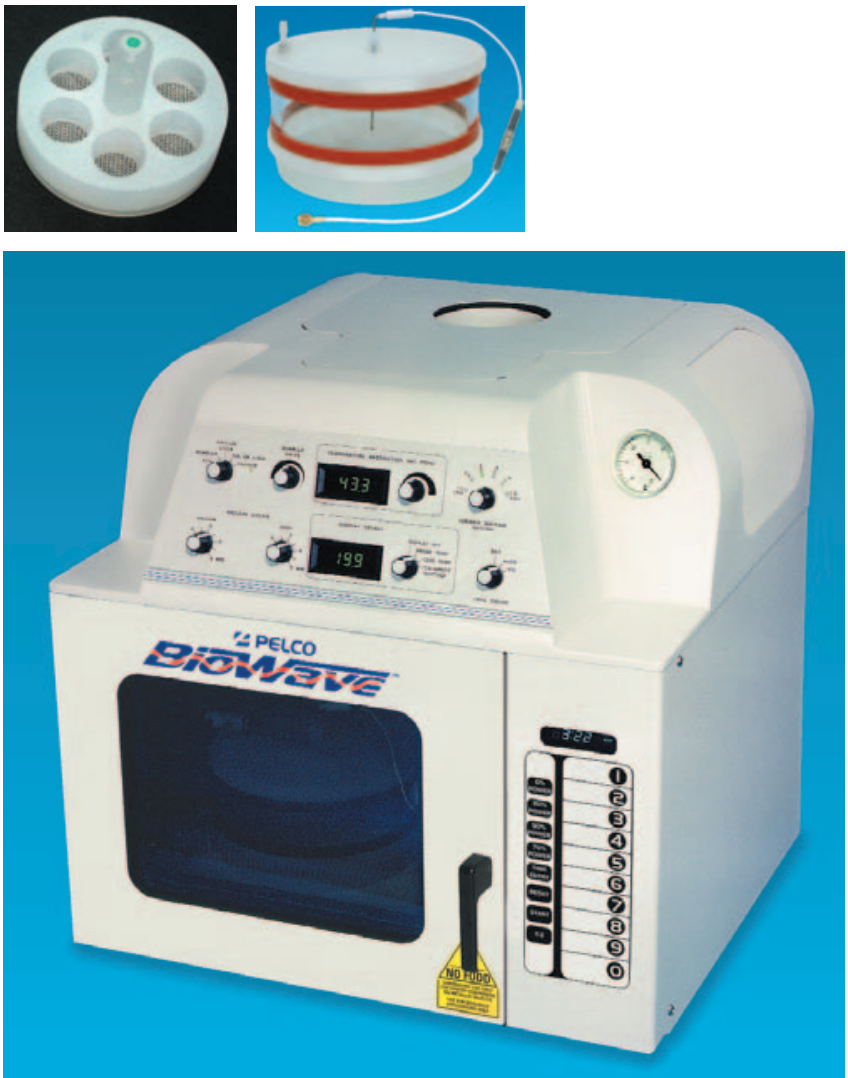

PELCO BioWave ${ }^{\circledR}$ Laboratory Tissue Processing System

\section{Vacuum}

The 3435 PELCO $^{\circledR}$ EM MW Vacuum Chamber holds vacuum down to 1 torr ( $1 \mathrm{~mm} \mathrm{Hg}, 1.33 \mathrm{mBar}$ ).

\section{Cooler than Ambient}

Our PELCO SteadyTemp ${ }^{m}$ will permit temperature control below ambient to be run through the PELCO ColdSpot ${ }^{\circledR}$, or allow for faster fixation or decalcification.

\section{Microwave Accessories}

designed for many steps, protocols and fields.

Other operating and safety features are included.
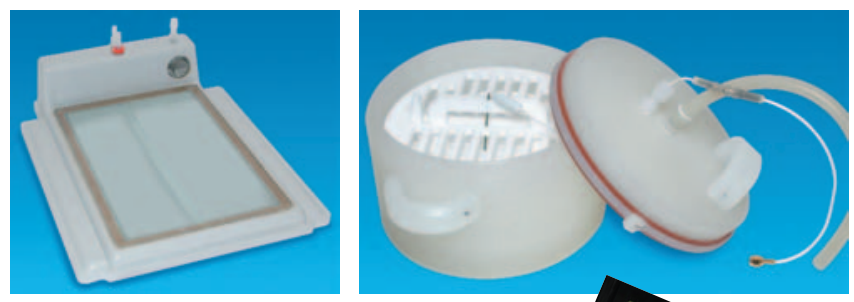

Micion

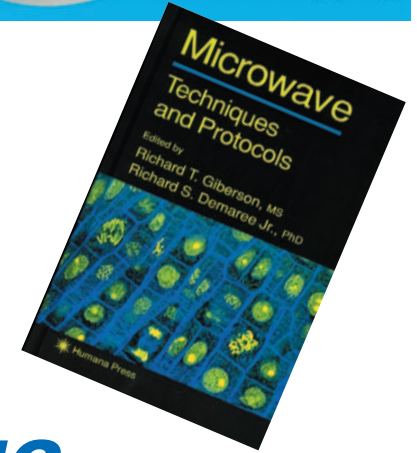

TED PELLA, INC.

Microscopy Products for Science and Industry 